\title{
Calibrating Screen Coordinates of Tabletop Display Using Shadow-Cursor
}

\author{
Makio Ishihara ${ }^{1}$ and Yukio Ishihara ${ }^{2}$ \\ 1 Fukuoka Institute of Technology, 3-30-1 Wajiro-higashi, Higashi-ku, Fukuoka, \\ 811-0295 Japan \\ m-ishihara@fit.ac.jp \\ http://www.fit.ac.jp/ m-ishihara/Lab/ \\ 2 Kyushu University, 3-1-1, Maidashi, Higashi-ku, Fukuoka, 812-8582 Japan \\ iyukio@redoxnavi.med.kyushu-u.ac.jp \\ http://hyoka.ofc.kyushu-u.ac.jp/search/details/K004222/english.html
}

\begin{abstract}
This manuscript conducts an experiment in usability of shadow cursor for calibrating screen coordinates of tabletop displays. Shadow cursor is a mouse cursor without any visual feedback or an imaginary mouse cursor. To calibrate the screen coordinates properly, users have to move the shadow cursor in an indicated direction correctly. The experiment shows that its accuracy is about \pm 5 degrees and the calibration is completed within about 700 milliseconds.
\end{abstract}

Keywords: shadow cursor, screen coordinates calibration, tabletop display, interaction design.

\section{Introduction}

A tabletop display is a horizontal display which multiple users sit down around and use together. The tabletop display is suitable well for collaborative work, so that it leads to a strong demand for a large screen space. One of key issues around large tabletop displays is how to make a pointing interface in the case that users cannot reach the contents by their own hands.

There have been three major ways of how a pointing interface is made for large tabletop displays. The first one uses sophisticated pointing sensors such as 6DOF magnetic ones, which may be used in CAVE systems. This often leads to expensive and cumbersome equipment. The second one uses a handheld camera to capture the quadrilateral of the entire screen so that the center of the camera performs a graphical cursor. Nintendo Wii remotes take this way. This way however works as far as the camera captures the quadrilateral of the entire screen. The third one uses invisible gray-code for the camera to capture not the entire screen but a position on the screen to locate it. Gray-code binary patterns appear on the screen in order then the temporal pattern at a position of the screen uniquely represents its own position. This way requires the screen to display invisible data to users like infrared one. 


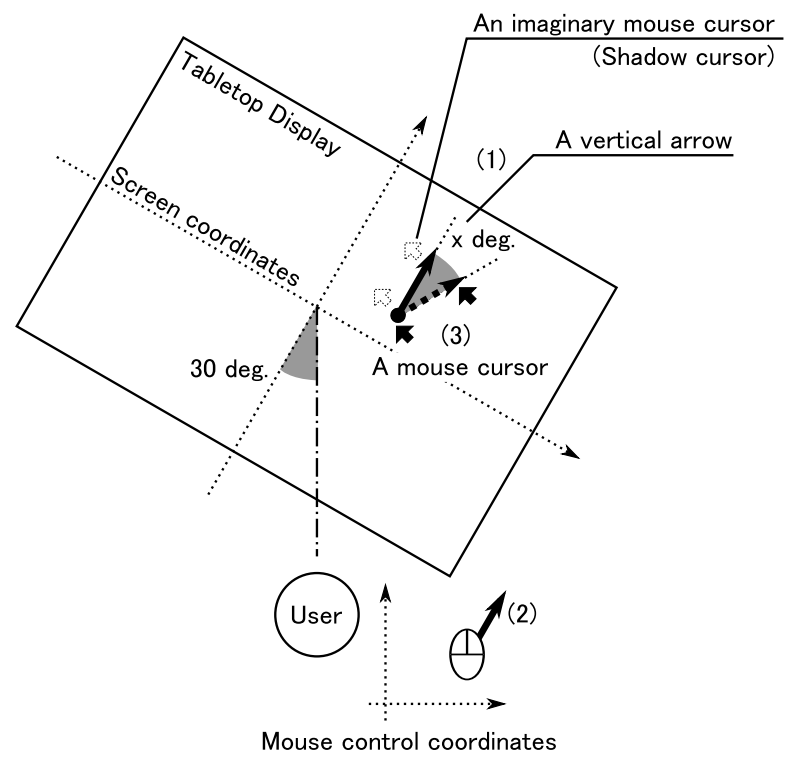

Fig. 1. Screen coordinates calibration with shadow cursor

Our pointing interface uses a common mouse. It is inexpensive and requires no infrared technique. One of major problems is that the coordinates of the screen (screen coordinates) are not always aligned with the coordinates of the mouse control space (mouse control coordinates). The first attempt to align the screen coordinates with the mouse control coordinates used a reflex in eye-hand coordination [1]. The experiments showed that the reflex would take about 4 seconds to align them and it was impractical. This manuscript reports on the second attempt which relies on the basic idea of shadow cursor. Shadow cursor allows users to perform a simple mouse-manipulation to align them, so that the calibration is expected to be completed in less than a second.

\section{Shadow Cursor}

A shadow cursor is an imaginary mouse cursor. Figure 1 shows how the shadow cursor works to align the screen coordinates with the mouse control coordinates. In the figure, there is the angular distance of 30 degrees between the screen coordinates and the mouse control coordinates. First, (1) a vertical arrow to the screen appears on it and (2) the user moves the mouse in the direction indicated by the vertical arrow and then (3) the mouse cursor will move at an angle of $\mathrm{x}$ degrees. In this case, x equals 30 degrees. At the step (3), the mouse cursor will move in the direction that the user does not expect, so that the mouse cursor is hopefully set invisible (invisible mouse cursor). The user needs to move the mouse without seeing the mouse cursor. In other words, the user needs to move 


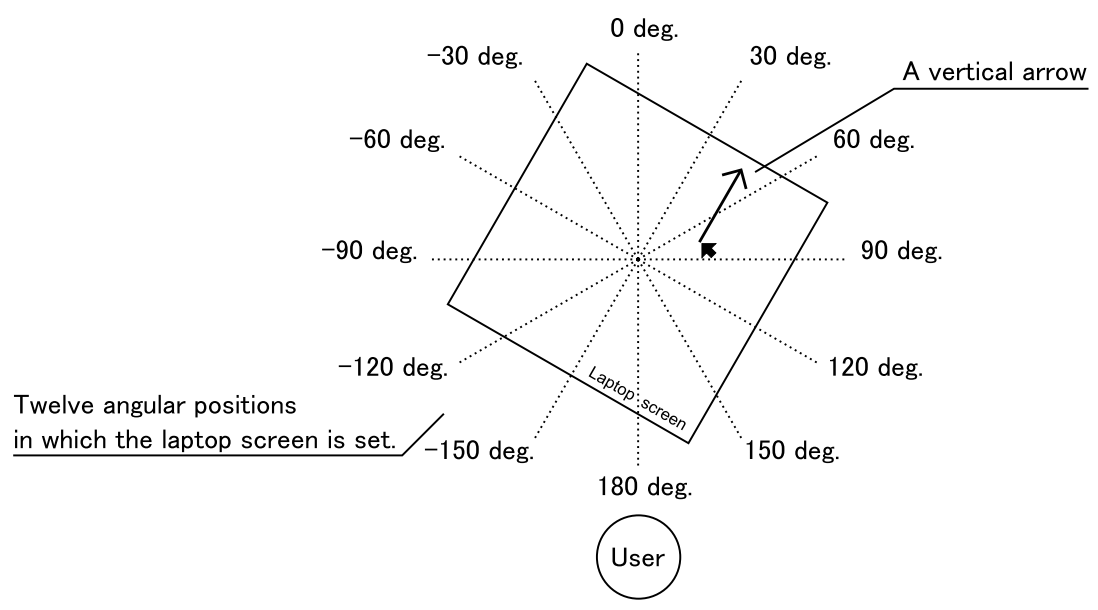

Fig. 2. Experiment settings

the mouse so that an imaginary mouse cursor (shadow cursor) moves in the direction indicated by the vertical arrow, where the user normally expects the invisible mouse cursor to move.

\section{Experiment in Usability}

We conducted an experiment in how accurately and fast the shadow cursor aligns the screen coordinates with the mouse control coordinates. Figure 2 shows the design of the experiment. The rectangle represents a laptop screen of Dell XPS L702X, which is $385 \mathrm{~mm}$ wide and $215 \mathrm{~mm}$ high (1600x900 pixels in resolution). The laptop has a quad-core processor of $2.40 \mathrm{GHz}$. It is also equipped with a graphics card of nVidia GT 555M. The laptop screen is set in an angular position of -150 to 180 at 30 degree intervals and a vertical arrow appears where the subject right-clicks on the screen. Then the mouse cursor becomes invisible and the subject is asked to move the shadow cursor in the direction indicated by the vertical arrow. After that, the angular distance between the screen coordinates and the mouse control coordinates is calculated when the invisible mouse cursor goes out of the circle of $10 \mathrm{~mm}$ radius as shown in Figure 3 . The screen coordinates are rotated by the observed angular distance.

There were 10 subjects between the ages of 22 and 36. All were right-handed and had an experience working with a mouse. Each subject had 10 trials for each angular position, resulting in 1200 trials. To avoid learning effects, the sequence of trials is in random order but it is the same between subjects.

10 subjects $\times 12$ angular positions $\times 10$ trials $=1200$ total trials 


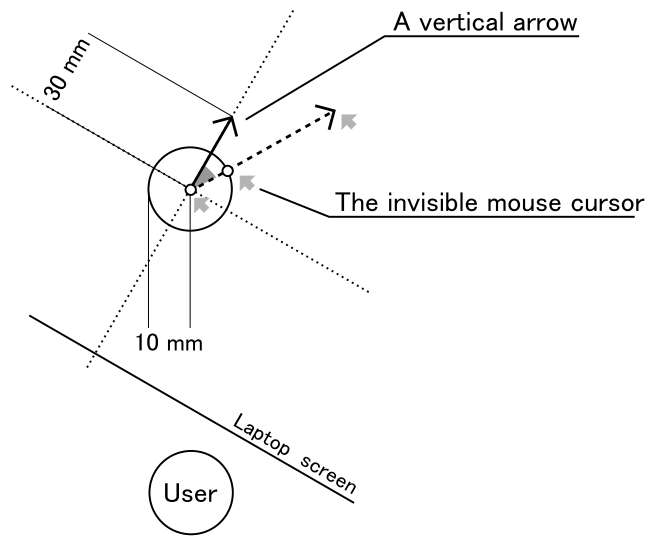

Fig. 3. Detecting the angular distance

\section{Results}

Figure 4 shows the result on calibration accuracy. The horizontal axis shows the initial angular position of screen and the vertical one shows accuracy of the observed angular position at each initial angular position across all the subjects. From the figure, there seems to be a certain positive correlation between the initial angular position and accuracy. This could be due to what the subjects' dominant hand is: They all are right-handed. There is also an increase in the center and two decreases around \pm 30 to 60 degrees, two increases again at both sides. This could be due to directions where users think they move the

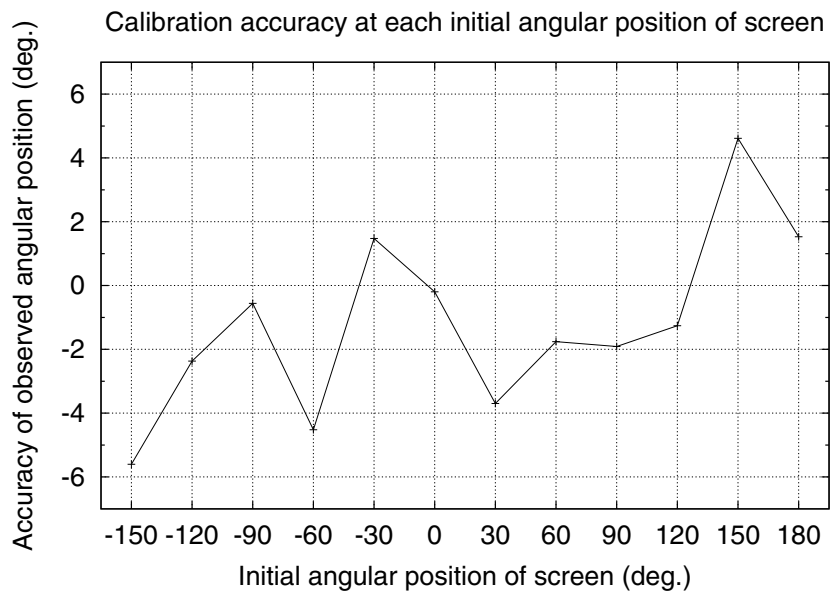

Fig. 4. Result of calibration accuracy 


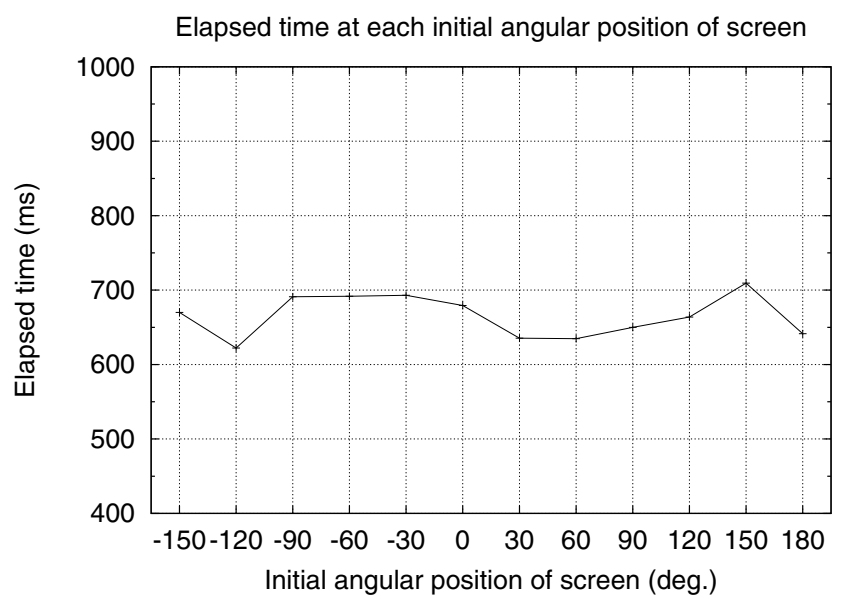

Fig. 5. Result of time efficiency

mouse comparatively easily: 0 and \pm 90 degrees. Analysis of variance shows that there is a significant impact of the initial angular position on calibration accuracy $[\mathrm{F}(11,1188)=4.91$ at $\mathrm{p}=0.001]$. The accuracy is however within about \pm 5 degrees. Figure 5 shows the result on time efficiency. The vertical axis shows the elapsed time to complete the calibration across all the subjects. From the figure, all the calibration is completed within about 700 milliseconds. The time efficiency is improved by $17 \%$ as compared to the previous way [1].

\section{Conclusions}

This manuscript conducted an experiment in usability of shadow cursor for calibrating screen coordinates of tabletop displays. The results showed that the shadow cursor presents a good performance in accuracy and time efficiency.

\section{Reference}

1. Ishihara, M., Ishihara, Y.: Calibrating coordinates of a tabletop display with a reflex in eye-hand coordination. IEICE Trans. on Information and Systems e93-d(10), 2862-2865 (2010) 